\title{
Residual Generator Selection for Fault Diagnosis of Hybrid Vehicle Powertrains
}

\author{
Christofer Sundström*, Erik Frisk*, and Lars Nielsen* \\ * Vehicular Systems, Dept. of Electrical Engineering, Linköping \\ University, SE-58183 Linköping, Sweden, \{csu,frisk,lars\}@isy.liu.se.
}

\begin{abstract}
The performance of a model based diagnosis system is affected by the selection of consistency relation in a set of equations with analytical redundancy in a non-linear system. To investigate aspects due to this, two diagnosis systems of a parallel hybrid truck are designed, and both static and dynamic issues are considered. A simplified vehicle model is used to exemplify how a unique expression for the residual generator can be found for one selection of consistency relation, but not for others, using the same set of equations. A simulation study using the entire vehicle model is made to investigate how the performance in the diagnosis system is affected when dynamic equations are either differentiated or integrated. The diagnosis systems are designed using structural analysis in combination with the algebraic expressions. One key result is that it is not trivial to find a computational order by hand that fulfills the predefined conditions on the computational sequence, and therefore systematic methods are valuable.
\end{abstract}

Keywords: parallel hybrid, consistency based diagnosis, structural analysis

\section{INTRODUCTION}

Diagnosis is used to detect and isolate faults in a system. There are several approaches to be used, and one of the more common is consistency based diagnosis (de Kleer et al., 1992) based on precompiled tests, or residual generators, as in Blanke et al. (2006). This work analyzes some fundamental choices when designing residual generators for dynamic systems. A basis for the analysis is a model of a hybrid electric powertrain in a heavy duty truck.

When hybridizing a vehicle, new components are added compared to a conventional vehicle, e.g. electric machines, battery, and power electronics. It is important to monitor these components due to safety issues since a fault in the high voltage system is fatal. It is also important to monitor the powertrain to avoid damaging components when a fault in another component has occurred. It is especially important to protect the battery that is expensive and may degrade fast if e.g. large power flows are used in the battery. High powers in the electrical components may be caused by a fault in e.g. the power electronics or the electric machine.

\subsection{Problem formulation}

A diagnosis system can be based on one or several residual generators, which are constructed from a set of model equations with analytical redundancy. In this investigation there is one more equation than unknowns in the set of equations used to construct the residual generators, and the equation not used in the just-determined part is the consistency relation. The selection of consistency relation affects the performance of the diagnosis system when a non-linear system is monitored. How the selection of the consistency relation affects the diagnosis performance is investigated by designing two diagnosis systems for a parallel hybrid truck. The investigation is done using a simplified model to exemplify some key issues and the entire vehicle model in a simulation study.

\section{BACKGROUND}

This section consists of two parts. First, when designing the diagnosis systems described in Section 3.4, a well known method called structural analysis is used. There are several different approaches and notations in the field, which are briefly described in Section 2.1. Secondly, a dynamic equation can in a computational sequence either be integrated or differentiated, and the notation used for this is given in Section 2.2.

\subsection{Structural analysis}

Structural analysis is an efficient tool when designing a model based diagnosis system. The analysis is based on a bipartite graph, including information about the variables that are included in each model equation. Based on this graph a Dulmage-Mendelsohn decomposition (Dulmage and Mendelsohn, 1958) gives information about what part of the model that is overdetermined and thereby can be monitored. There are several efficient tools available to find subsets of the model with analytical redundancy, and some of these are discussed and compared in Armengol et al. (2009). Overdetermined sets are of special interest since they are used to construct residuals, and are denoted e.g. ARRs (Cassar and Staroswiecki, 1997), possible conflicts (Pulido and Gonzalez, 2004), and MSOs (Krysander et al., 2008).

The first developed algorithms finding sets of equations with analytical redundancy one (MSOs) do not consider how to find the algebraic expressions for the residual generators. Recent algorithms that consider invertibility and how differential equations are solved in the residual generators have been developed, see e.g. de Flaugergues et al. (2009). These algorithms investigate the properties of the MSOs, but do not provide the user information about which consistency relations that are possible to use to achieve wanted properties of the residual generators. Svärd and Nyberg (2010) analyzes the characteristics of the system for every possible consistency relation. This is done by first using structural analysis to find the overdetermined sets of equation, and the computational order of the unknowns is found using Dulmage-Mendelsohn decomposition after a consistency relation is selected. The algebraic expressions are then used in the algorithm to analyze if predefined constraints of the residual generator is fulfilled. Such 


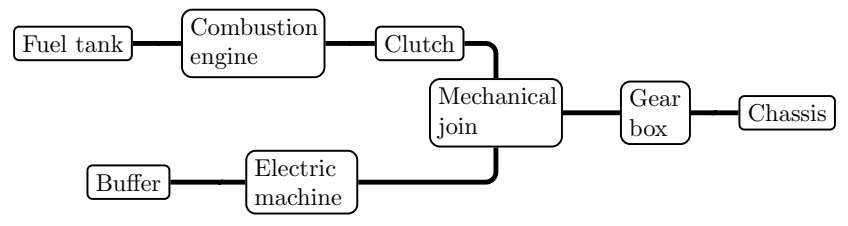

Fig. 1. The modeled truck is a parallel hybrid with the connection of the electrical and conventional parts of the powertrain between the clutch and the gearbox.

constraints may e.g. be that differential equations only can be solved by differentiating or integrating a signal, and that a unique residual generator is to be found. These properties may vary depending on the selection of the consistency relation in a set of equations, and therefore all possible residual generators are to be investigated. This methodology is used in this paper.

\subsection{Dynamic equations}

The system used in this investigation is based on model equations, $g$, including dynamics

$$
g\left(x_{1}, \dot{x}_{1}, x_{2}, z\right)=0
$$

where $x_{1}$ is a vector of unknown dynamic variables, $x_{2}$ is a vector of unknown algebraic variables, and $z$ is a vector of known signals. The relation between a variable $x_{1, i} \in x_{1}$ and $\dot{x}_{1, i}$ is given in the dynamic equation

$$
\frac{d}{d t} x_{1, i}=\dot{x}_{1, i}
$$

that can be used in a computational sequence using two different methods or computational causalities, here causality is used for short (Frisk et al., 2010):

derivative causality is when $x_{1, i}$ is differentiated to obtain $\dot{x}_{1, i}$, i.e. $\dot{x}_{1, i}:=\frac{d}{d t} x_{1, i}$

integral causality is when $\dot{x}_{1, i}$ is integrated to obtain $x_{1, i}$, i.e. $x_{1, i}:=\int \dot{x}_{1, i} d t+C$, where $C$ is the initial value of $x_{1, i}$.

mixed causality is when a system can be solved using both derivative and integral causality

\section{SIMULATION ENVIRONMENT}

The simulation environment consists of a vehicle model and a diagnosis system. Parts of the environment will be described below and the entire description is given in Sundström (2011).

\subsection{Vehicle model}

The modeled vehicle is a long haulage electric parallel hybrid truck configured as in Figure 1. The models of the components in the vehicle are based on the model library developed in the Center for Automotive Propulsion Simulation (CAPSim, 2009), and the QSS toolbox (Guzzella and Amstutz, 1999). Modifications to these models are carried out to model a truck instead of a passenger car, and to include the possibility to induce faults in the models as well as to add sensor noise. The models in the electric components and the clutch are given below since these are key components in the later described diagnosis systems.

Battery The battery is modeled using a Thévenin equivalence circuit, i.e. a voltage source and an inner resistance connected in series. The capacity and nominal voltage are $9 \mathrm{kWh}$ and $256 \mathrm{~V}$, respectively.
Table 1. The intensity of the noise added to the sensor signals in the model.

\begin{tabular}{cc} 
Sensors & Noise power \\
\hline$I_{b, \text { sens }}$ & 0.01 \\
$I_{e m, \text { sens }}$ & 0.01 \\
$U_{e m, \text { sens }}$ & 0.01 \\
$\omega_{i c e, \text { sens }}$ & 0.01 \\
$\omega_{g b, \text { sens }}$ & 0.01 \\
\hline
\end{tabular}

Electric machine The electric machine is able to convert electric power to mechanical power and vice verse. A voltage, $U_{e m}$, is applied on the component, resulting in a torque on the outgoing shaft. The torque, $T_{e m}$, is proportional to the armature current, $I_{e m}$

$$
\begin{aligned}
I_{e m} & =\frac{U_{e m}-k_{i} \omega_{e m}}{R_{e m}} \\
T_{e m} & =I_{e m} k_{a}
\end{aligned}
$$

where $\omega_{e m}$ is the angular speed of the machine (Guzzella and Sciarretta, 2007). The model is parametrized as a $33 \mathrm{~kW}$ DC machine with constant magnetic flux. The parameter values of the resistance, $R_{e m}$, torque constant, $k_{a}$, and speed constant, $k_{i}$, set to $0.044 \Omega, 0.50 \mathrm{Nm} / \mathrm{A}$, and $0.51 \mathrm{Vs} / \mathrm{rad}$, respectively. In an ideal machine, $k_{i}$ and $k_{a}$ are equal, and are defined by $K \phi$, where $K$ is a machine constant that depends on the design parameters of the machine, and $\phi$ is the magnetic flux produced by the stator. This model can be used to represent a permanent magnet synchronous machine, that is a common machine type in HEVs due to its high efficiency (Chau et al., 2008).

Power electronics The model of the power electronics is assumed to be an ideal component

$$
P_{b}=P_{e m} \Longleftrightarrow I_{b} U_{b}=I_{e m} U_{e m}
$$

where $P_{b}$ and $P_{e m}$ are the electrical power from the battery and motor, and $U_{b}$ and $I_{b}$ the battery voltage and current.

Clutch There is a model of the clutch to handle starts and gear shifts. When the clutch is fully engaged or disengaged, the clutch is modeled as an ideal component, but the model is more complex when there is slip in the clutch.

\subsection{Sensors}

The sensors available for the diagnosis system used in the simulation study are given in Table 1 . The measurement signal, $y$, is given by the noise free signal, $y^{*}$ added with noise, $\tilde{\nu}$

$$
y=y^{*}+\tilde{\nu}
$$

To simulate measurement noise a Band-Limited White Noise block in Simulink is used with the noise powers according to Table 1.

\subsection{Faults}

To model that the battery, power electronics, or the electric machine may break down, two parameter values and two voltages in these models have the possibility to be modified. Note that these faults are only examples of how a fault in these components can be represented in the model. The following modifications of the signals are introduced to model the faults where the nominal signals are denoted by the superscript nom:

$$
\begin{aligned}
f_{e m, k a} & : k_{a}=\left(1+f_{e m, k a}\right) k_{a}^{n o m} \\
f_{e m, R}: & R=\left(1+f_{e m, R}\right) R_{e m}^{n o m} \\
f_{p e} & : U_{e m}=\left(1+f_{p e}\right) U_{e m}^{n o m} \\
f_{b, s c}: & U_{b}=\left(1+f_{b, s c}\right) U_{b}^{n o m}
\end{aligned}
$$

where $f_{b, s c}$ models that all cells in the battery are not used due to an internal short circuit, $f_{p e}$ is a fault in the 
Table 2. Values of the faults induced in the model. The voltage $U_{e m}$ is between $0-200 \mathrm{~V}$.

\begin{tabular}{rl} 
Faults & Value \\
\hline$f_{\text {em }, k i}$ & -0.5 \\
$f_{\text {em, }}$ & -0.5 \\
$f_{p e}$ & -0.5 \\
$f_{b, s c}$ & -0.5 \\
$f_{\text {em }, U, s e n s}$ & $20 \mathrm{~V}$ \\
\hline
\end{tabular}

power electronics, and $f_{e m, k a}$ and $f_{e m, R}$ are two faults in the electric machine.

The voltage sensor in the electric machine is monitored in the diagnosis systems. A fault in the sensor is modeled as an offset fault

$$
U_{\text {em,sens }}=U_{\text {em }}+f_{\text {em }, U, \text { sens }}
$$

When a fault is induced in the model, the value of the fault is given in Table 2 .

\subsection{Diagnosis systems}

The diagnosis systems this investigation is based on are described in Sundström (2011), where they are denoted Diagnosis systems 2 and 3. Diagnosis system 2 consists of four tests and theoretically achieves full isolability. This diagnosis systems is compared with a modified diagnosis system in a simulation study in Section 5, while one test in System 3 is the basis for the study about algebraic loops in Section 4. This test consists of 35 of the overall 46 model equations of the vehicle, and the residual generator has two solutions due to a nonlinear algebraic loop. To investigate why no unique solution is found for this residual generator a simplified example based on seven equations is used.

Below two key issues are briefly described; how the dynamics in the residual generators are handled, and that the model used in the diagnosis systems are not valid in all operating modes.

Dynamic residual generators Two of the four tests in the Diagnosis system 2, that is used in the simulation study, include dynamics since the connection between the resulting torque on the wheels and the acceleration of the vehicle is used in the corresponding residual generators. The residual generators are presented in Sundström (2011) and are expressed in the form

$$
\tilde{r}=a \dot{\omega}_{g b}+b
$$

where $\omega_{g b}$ is the outgoing angular velocity from the gearbox, $a$ is the sum of the effective inertia and mass of the vehicle, and $b$ is the net torque acting on the wheels. By filtering $\tilde{r}$ in (7a) the residual $r$ is obtained

$$
r=\frac{\alpha}{p+\alpha} \tilde{r}=\frac{\alpha}{p+\alpha}\left(a \dot{\omega}_{g b}+b\right)
$$

Now, it is possible to compute $r$ in $(7 \mathrm{~b})$, without calculating a differentiated signal using a transformation. The conditions for this to be possible is that $a$ is a constant and $b$ a function of known signals, and the residual is filtered as in (7b) (Frisk and Nyberg, 2001). Using the state

$$
\Gamma=r-\alpha a \omega_{g b}
$$

we obtain that the residual generator in $(7 \mathrm{~b})$ can be expressed as

$$
\begin{aligned}
\dot{\Gamma} & =-\alpha \Gamma-\alpha^{2} a \omega_{g b}+\alpha b \\
r & =\Gamma+\alpha a \omega_{g b}
\end{aligned}
$$

Model not valid The model of the clutch used in the diagnosis systems is only valid when the clutch is fully released. This results in that when the clutch is disengaged or there is slip in the clutch, corresponding test quantities
Table 3. Permuted structural model of the system given in (8) except $e_{1}$ that is chosen to the consistency relation. Equations $e_{2}$ and $e_{3}$ form an algebraic loop for $I_{e m}$ and $U_{e m}$.

\begin{tabular}{c|cccccc} 
& $\omega$ & $U_{b}$ & $\dot{\omega}$ & $I_{b}$ & $U_{e m}$ & $I_{e m}$ \\
\hline$e_{7}$ & $\mathrm{X}$ & & & & & \\
$e_{6}$ & & $\mathrm{X}$ & & & & \\
$e_{5}$ & $\mathrm{X}$ & & $\mathrm{X}$ & & & \\
$e_{4}$ & & $\mathrm{X}$ & & $\mathrm{X}$ & & \\
$e_{3}$ & & $\mathrm{X}$ & & $\mathrm{X}$ & $\mathrm{X}$ & $\mathrm{X}$ \\
$e_{2}$ & $\mathrm{X}$ & & & & $\mathrm{X}$ & $\mathrm{X}$
\end{tabular}

are not updated and no faults are to be detected. There are also demands on that a gear has to be selected and the voltage to the electric machine is to be above a threshold for the test quantities to be updated.

\section{ALGEBRAIC LOOPS}

Algebraic loops, as well as that the unknown variables are not invertible, may occur in the just-determined parts of the MSOs. There are several numerical and analytical solving methods available to solve algebraic loops. Linear algebraic loops are e.g. easily solved, but other algebraic loops may demand a large computational effort to solve and a solution is not always obtained. Maple is here used to investigate if it is possible to analytically find a unique expression for the residual generator.

The possibility to find a unique residual generator given a set of equations in an MSO varies with the chosen consistency relation. This is exemplified with the reduced set of model equations in the MSO used in one test in Diagnosis system 3, as indicated in Section 3.4

$$
\begin{aligned}
e_{1}: & T_{e}+\underbrace{k_{a} I_{e m}}_{T_{e m}}-T_{l}(\omega)-J_{t o t} \dot{\omega}=0 \\
e_{2}: & \frac{U_{e m}-\omega k_{i}}{R_{e m}}-I_{e m}=0 \\
e_{3}: & I_{b} U_{b}-I_{e m} U_{e m}=0 \\
e_{4}: & U_{o c}-U_{b}-R_{b} I_{b}=0 \\
e_{5}: & \frac{d}{d t} \omega-\dot{\omega}=0 \\
e_{6}: & U_{b}-y_{1}=0 \\
e_{7}: & \omega-y_{2}=0
\end{aligned}
$$

where $\omega$ is an angular speed, $J_{\text {tot }}$ the inertia of the vehicle, $U_{o c}$ and $R_{b}$ the open source voltage and the inner resistance in the battery, $T_{l}$ the lumped torque due to losses in the vehicle, $T_{e}$ the torque from the engine, and $y_{1}$ and $y_{2}$ are sensor signals. The torques $T_{e}$ and $T_{l}$ are in this example assumed to be known.

If $e_{1}$ is selected as the consistency relation, the permuted structural model of the just-determined part, i.e. $\left\{e_{2}-e_{7}\right\}$, is given in Table 3 . The corresponding computational order would be:

$$
\begin{aligned}
\mathcal{C}=\left(\{\omega\},\left\{e_{7}\right\}\right), & \left(\left\{U_{b}\right\},\left\{e_{6}\right\}\right),\left(\{\dot{\omega}\},\left\{e_{5}\right\}\right), \\
& \left(\left\{I_{b}\right\},\left\{e_{4}\right\}\right),\left(\left\{I_{e m}, U_{e m}\right\},\left\{e_{2}, e_{3}\right\}\right)
\end{aligned}
$$

indicating that $\omega$ is computed from $e_{7}, U_{b}$ is computed from $e_{6}$ and so forth. The pair $\left(\left\{I_{e m}, U_{e m}\right\},\left\{e_{2}, e_{3}\right\}\right)$ indicates that there is an algebraic loop, that also can be seen in Table 3. This loop has the non unique solution

$$
I_{e m}=-\frac{\omega k_{i}}{2 R_{e m}} \pm \sqrt{\left(\frac{\omega k_{i}}{2 R_{e m}}\right)^{2}+\frac{I_{b} U_{b}}{R_{e m}}}
$$

If one of $e_{2}$ or $e_{3}$ is used as consistency relation instead of $e_{1}$, there is no algebraic loop in the just-determined part. Since the variables in the substitution chain are invertible, 
a unique residual generator can therefore be expressed. The computational order of the unknown variables if e.g. $e_{2}$ is used as the consistency relation will be

$$
\begin{aligned}
& \mathcal{C}=\left(\{\omega\},\left\{e_{7}\right\}\right),\left(\left\{U_{b}\right\},\left\{e_{6}\right\}\right),\left(\{\dot{\omega}\},\left\{e_{5}\right\}\right), \\
&\left(\left\{I_{b}\right\},\left\{e_{4}\right\}\right),\left(\left\{I_{e m}\right\},\left\{e_{1}\right\}\right),\left(\left\{U_{e m}\right\},\left\{e_{3}\right\}\right)
\end{aligned}
$$

Note that since consistency based diagnosis is used, it is possible to construct a test that is based on a residual generator with several solutions. As long as at least one of the possible residuals is close to zero, the test will not react

$$
\left|r\left(t_{k}\right)\right|=\min \left\{\left|r_{1}\left(t_{k}\right)\right|,\left|r_{2}\left(t_{k}\right)\right|, \ldots,\left|r_{i}\left(t_{k}\right)\right|\right\}, i \geq 2
$$

However, the computational complexity of the system increases if more than one residual are to be evaluated in a test.

\subsection{Series wound electric machine}

In the example above the magnetic field, $\phi$, created by the stator or armature is assumed to be constant. This is the case in permanent magnet synchronous machines, that is the machine type mainly used for vehicle propulsion in hybrid electric vehicles. But if a series wound machine is used instead, that e.g. is used in starter motors (Hambley, 2005), $e_{1}$ and $e_{2}$ in (8) are modified to

$$
\begin{aligned}
& e_{1}: \quad T_{e}+\underbrace{k_{a} I_{e m}^{2}}_{T_{e m}}-T_{l}(\omega)-J_{t o t} \dot{\omega}=0 \\
& e_{2}: \quad \frac{U_{e m}}{R_{e m}+\omega k_{i}}-I_{e m}=0
\end{aligned}
$$

due to that $\phi$ increases linearly with the current in the rotor and stator according to

$$
\phi=k I_{e m}
$$

The same variables are included in each equation in (8) and (12), and therefore the structural models are the same for the two systems. It is however not possible to chose a consistency relation that results in a unique expression for the residual generator in the later system. This is due to that the algebraic loop in $e_{2}$ and $e_{3}$ adds the constraint that one of these equations needs to be used as consistency relation. The current $I_{e m}$ is then to be calculated using $e_{1}$, but since $I_{e m}$ is not longer invertible in $e_{1}$, it is not possible to find a unique residual generator.

\section{INTEGRAL AND DERIVATIVE CAUSALITY}

In this section differential equations are considered. It is not possible to state that one of integral, derivative, or mixed causality always performs best and therefore is preferable. In general it is not preferable to differentiate a noisy signal, and not to integrate a signal in a diagnosis system where an offset occurs since this will lead to drift in the integrator.

In this investigation two diagnosis systems are compared, one based on mixed causality and one on integral causality. The basis for these diagnosis systems is Diagnosis system 2 that briefly is described in Section 3.4. Test 3 and Test 4 used in the system include dynamics and are therefore handled here. In the original system, mixed causality is used in these two tests. The mass of the fuel consumed, $m_{f}$, is solved by using integral causality

$$
m_{f}=\int \dot{m}_{f} d t
$$

and a similar equation to $e_{1}$ in (8) is used as consistency relation, where $w_{g b}$ is differentiated to compute $\dot{\omega}_{g b}$, leading to that derivative causality is used. For the computation the reformulation in (7) is used.

\subsection{MCDS and ICDS}

The diagnosis system described above where two of the tests are based on mixed causality, is denoted mixed causality diagnosis system, or MCDS for short. The MCDS is to be compared with a system where the following constraints are to be fulfilled:

- integral causality is used

- the set of equations in the just-determined parts of the MSOs are globally invertible

This diagnosis system is denoted integral causality diagnosis system, or ICDS for short.

\subsection{Methodology to construct ICDS}

The algorithm used to find the ICDS is based on the algorithm described in Svärd and Nyberg (2010) and briefly recalled in Section 2.1. In the original algorithm only the just-determined part of the MSO is considered, i.e. the consistency relations is not included in the analysis determining the properties of the residual generators. When designing ICDS also the consistency relation is included in the analysis. This only affects the constraint on how differential equations are to be solved, since all unknowns are calculated in the just-determined part and therefore invertibility is not an issue in the consistency relation. The constraint in Section 5.1 regarding differential equations states that derivative causality is not to be used in ICDS. One of the constraints on the analyzed set of equations is that, without loss of generality, a differentiated variable may only occur once in the system. Therefore, if a differentiated signal is included in the consistency relation, the output from the original algorithm might be that only integrating causality is used. But if there is a differentiated variable in the consistency relation, a signal must be differentiated since it is only known in its undifferentiated form from the just-determined set of equations. Therefore derivative causality is used in the residual generator in such a case.

\section{RESULTS AND DISCUSSION}

One result from this study is the outcome from the analysis of the diagnosis systems regarding algebraic loops and different ways of computing differential equations. The other main result is a simulation study comparing the performance in the mixed causality diagnosis system and the integral causality diagnosis system defined in Section 5.1.

\subsection{Selection of consistency relations used in ICDS}

Given the five sensors available in the diagnosis systems used in the study about how differential equations are solved, 79 MSOs are found. Each equation in an overdetermined part can potentially be selected as the consistency relation used to construct the residual generator for that set of equations. For all MSOs there are 2162 residuals generators to be investigated. In Figure 2 the number of model equations in each MSO is shown, but also how many equations that can be selected as consistency relation to fulfill the requirements in ICDS, i.e. integral causality and global invertibility. In MSOs 15-79 there is only a small fraction of the equations that can be used as consistency relations in the ICDS in order to fulfill these constraints. These MSOs include an equation similar to $e_{1}$ in (8), but computes the torques and angular speeds in different ways. There are five MSOs that do not have any consistency relation that fulfills the requirements.

The four tests used in the diagnosis system based on mixed causality, are included in the set of 74 MSOs that 


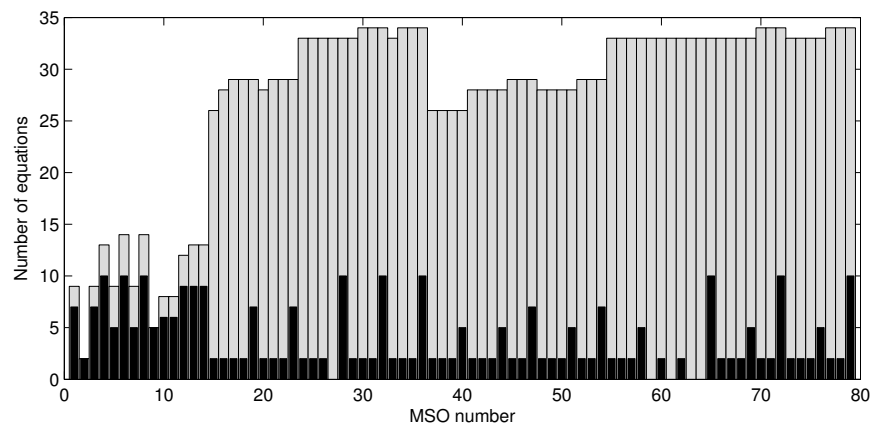

Fig. 2. The gray bars indicate the number of equations in each MSO in the diagnosis systems, and the black bars indicate the number of equations that can be selected to consistency relations in each MSO to fulfill the constraints in the ICDS. The number of MSOs and equations in MCDS and ICDS are the same, since the same vehicle model and sensor configuration is used.

fulfills the constraints for ICDS given in Section 5, for at least one selection of consistency relation. The residual generators used in Test 1 and Test 2 in the MCDS fulfills the constraints for ICDS using the selected consistency relation, and are therefore used unchanged in the new diagnosis system. In the MSOs used in Test 3 and Test 4 in MCDS, there are two equations that can be selected as the consistency relation in ICDS. These are

$$
\begin{aligned}
\omega_{g b} & =\omega_{g b, \text { sens }} \\
\omega_{w} & =\frac{\omega_{g b}}{u_{f}}
\end{aligned}
$$

for both tests where $\omega_{w}$ and $\omega_{g b}$ are the angular speeds at the wheels and the outgoing shaft of the gearbox, and $u_{f}$ is the gear ratio in the final gear. When using one of these consistency relations, $\dot{\omega}_{w}$ is calculated using corresponding equation to $e_{1}$ in 8 . The angular velocity $\omega_{w}$ is calculated by integrating this signal

$$
\omega_{w}=\int \dot{\omega}_{w} d t
$$

to be used in the consistency relation. In the two residual generators, (15) is used as consistency relation and $\omega_{w}$ in (17) is multiplied with the final gear to calculate $\omega_{g b}$.

The algebraic loop for $I_{e m}$ and $U_{e m}$ considered in Section 4 is not an issue in these two residual generators, since $U_{e m}$ is known without using any of $e_{2}$ and $e_{3}$ in (8). The required voltage from the power electronics is known in Test 3 , and the sensor measuring $U_{e m}$ is available in Test 4 .

\subsection{Simulation study}

To evaluate how the performance of the diagnosis systems is affected when differential equations are computed in different ways, simulations of a long haulage truck are carried out. The faults are induced one by one in the simulations, and the driving cycle used is FTP75. Since Test 1 and Test 2 do not differ in the two diagnosis systems, the simulation results for these tests are not presented.

The test quantities, $T$, are based the CUSUM algorithm (Page, 1954) given by

$$
T\left(t_{k}\right)=\max \left\{0, T\left(t_{k-1}\right)+\left|r\left(t_{k}\right)\right|-\nu\right\}
$$

Two parameters are used in the algorithm; $\nu$ that is an offset parameter that corresponds to the model uncertainty and noise in the residuals, and a threshold $J$. Normalized test quantities, $T_{\text {norm }}$, are calculated using

$$
T_{\text {norm }}=\frac{T}{J}
$$

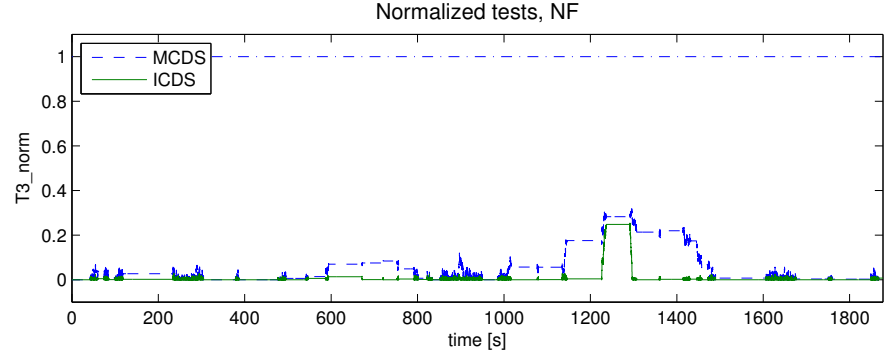

Fig. 3. The normalized test quantities in Tests 3 in the two diagnosis systems based on mixed and integral causality when the system is fault free. The tests react if the signal is above one. No false detection occurs in the diagnosis systems and the test quantities are well below the thresholds. Similar results are achieved in Tests 4 .

and the test alarms if $T_{\text {norm }}>1$. The parameters $\nu$ and $J$ in CUSUM are designed to achieve approximately the same maximum value of $T_{n o r m}$ in the tests in the fault free case. There are several combinations of $\nu$ and $J$ to achieve this; a smaller value of $\nu$ requires a larger value of $J$ and vice verse.

Initialization of states The state in the transformation used in MCDS is reinitialized when the model used in the diagnosis system is getting valid as described in Section 3.4. The state $w_{w}$ calculated from (17) is reinitialized in the residual generators used in ICDS. This is done by using a filtered sensor signal scaled with the final gear

$$
\omega_{w}\left(t_{0}\right)=\frac{1}{\tau_{w} s+1} \frac{\omega_{g b, \text { sens }}\left(t_{0}\right)}{u_{f}}
$$

where $t_{0}$ is the time when the residual generator is getting valid, and $u_{f}$ is the final gear.

In the initialization of the states in both MCDS and ICDS, it is assumed that the monitored system is fault free and the residual is zero. If the equations used in the expression for the signal to be integrated are inconsistent with the monitored system, the integrated signal will drift from the true value. The test quantity is therefore not updated during the first 10 seconds after a test has been valid to be able to detect the fault, and not use a residual that is close to zero even though the estimation of the signal to be integrated is inconsistent.

Simulation results In Figure 3, the normalized test quantities, $T_{\text {norm }}$ from the simulations in the fault free case for both diagnosis systems are presented. All test quantities are well below one, and no false alarm occurs in the simulations.

The normalized test quantities for Tests 4 (both MCDS and ICDS) when $f_{\text {em, } U \text {,sens }}$ occurs are given in Figure 4. Tests 3 do not react on this fault, as expected, and the simulation results for these tests are not included. Figure 5 shows $T_{n o r m}$ for Tests 3 when the resistance in the electric machine is modified.

All five fault modes are fully isolated in the ICDS, and all except the fault in the voltage sensor in the electric machine in the MCDS. The reason that $f_{\text {em }}$, sens is not isolated from a fault in the power electronics, is that Test 4 does not react as expected in the MCDS on this fault (see Figure 4). The normalized test quantity when there is a fault in the voltage sensor is almost one, and a different selection of parameters in the CUSUM algorithm possibly achieves full isolability for the MCDS. 


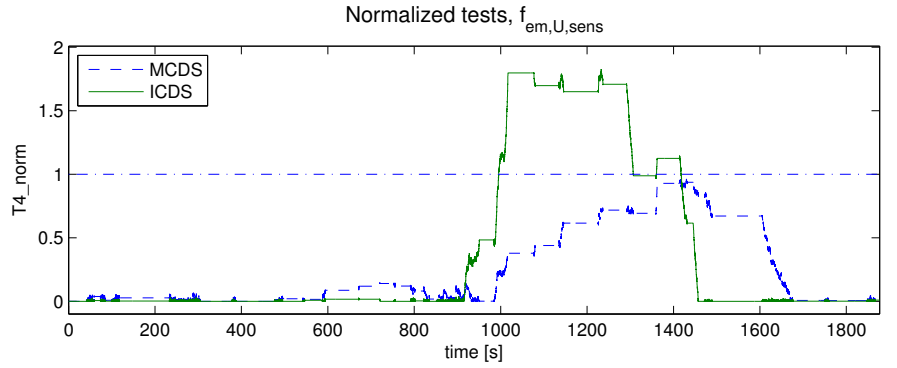

Fig. 4. The normalized test quantities when a fault is induced in the voltage sensor in the electric machine after 400 seconds. The test quantity in the MCDS is almost above the threshold, but the test does not alarm. The test in the ICDS reacts approximately 600 seconds after the fault is induced.

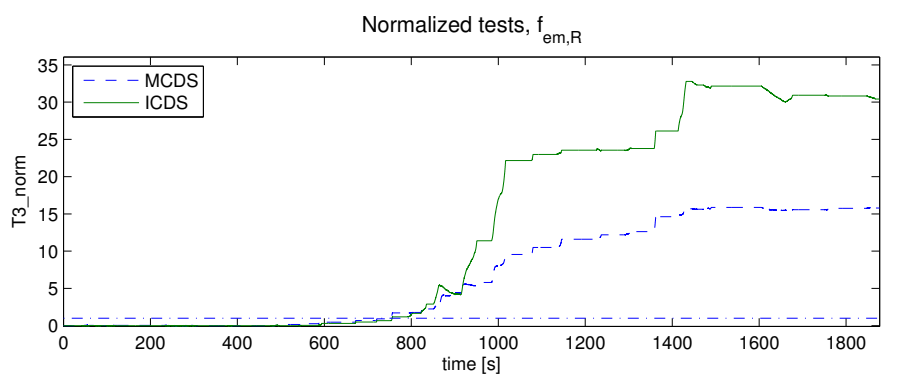

Fig. 5. The normalized test quantities when $f_{e m, R}$ is induced in the model after 400 seconds. Both diagnosis systems react about 350 seconds after the fault is induced in the simulation model. The magnitude of the test quantity in the ICDS is almost twice that of the MCDS at the end of the simulation.

\section{CONCLUSION}

The selection of consistency relation affects the performance of the diagnosis system. Predefined constraints are set up when selecting MSOs and consistency relations to be used, but it is not obvious how to select these constraints to achieve as good performance as possible in the diagnosis system.

It is shown, by using a realistic example, that algebraic loops in residual generators can be avoided for certain selections of consistency relations, but not for others. When the electric machine type is changed from permanent magnet to series wound, it was no longer possible to find a unique residual generator. This is due to that all unknowns are no longer globally invertible if the algebraic loop is to be avoided.

By including the consistency relation in the analysis of the properties of the residual generators, it is possible to avoid the use of the transformation of the residual generators that is used in MCDS. In the two tests in the diagnosis system based on integral causality used in the simulation study, there are only two of approximately 30 equations that can be selected to consistency relations to fulfill the stated constraints. It can be stated that it is not trivial to find consistency relations in large systems that fulfills predefined constraints on the diagnosis system. Systematic methods are therefore valuable.

In this investigation, the parameters used in the model are identical to those used in the vehicle model, and therefore it is reasonable to assume that integral causality is preferable, since sensor noise is included in the models of the sensors. The simulation study indicates that this is the case. When a fault is induced in the vehicle model, the test quantities generally react better in the system based on integral causality compared to the system based on mixed causality.

\section{REFERENCES}

Armengol, J., Bregon, A., Escobet, T., Gelso, E.R., Krysander, M., Nyberg, M., Olive, X., Pulido, B., and Trave-Massuyes, L. (2009). Minimal structurally overdetermined sets for residual generation: A comparison of alternative approaches. In Proceedings of IFAC Safeprocess'09. Barcelona, Spain.

Blanke, M., Kinnaert, M., Lunze, J., and Staroswiecki, M. (2006). Diagnosis and Fault-Tolerant Control. Springer, 2nd edition.

CAPSim (2009). http://www.capsim.se.

Cassar, J. and Staroswiecki, M. (1997). A structural approach for the design of failure detection and identification systems. In Proceedings of IFAC Control of Industrial Systems. Belfort, France.

Chau, K., Chan, C., and Liu, C. (2008). Overview of permanent-magnet brushless drives for electric and hybrid electric vehicles. Industrial Electronics, IEEE Transactions on, 55(6), 2246-2257.

de Flaugergues, V., Cocquempot, V., Bayart, M., and Pengov, M. (2009). Structural analysis for fdi: a modified, invertibility-based canonical decomposition. In Proceedings of the 20th International Workshop on Principles of Diagnosis, 59-66.

de Kleer, J., Mackworth, A., and Reiter, R. (1992). Characterizing diagnoses and systems. Artificial Intelligence, 56(2-3), 197-222.

Dulmage, A. and Mendelsohn, N. (1958). Coverings of bipartite graphs. Canadian J. of Mathematics, 10, 517534.

Frisk, E., Bregon, A., Åslund, J., Krysander, M., Pulido, B., and Biswas, G. (2010). Diagnosability analysis considering causal interpretations for differential constraints. In Proceedings of the 21th International Workshop on Principles of Diagnosis. Portland, USA.

Frisk, E. and Nyberg, M. (2001). A minimal polynomial basis solution to residual generation for fault diagnosis in linear systems. Automatica, 37(9), 1417-1424.

Guzzella, L. and Amstutz, A. (1999). CAE tools for quasistatic modeling and optimization of hybrid powertrains. IEEE Trans. on Vehicular Tech., 48(6), 1762-1769.

Guzzella, L. and Sciarretta, A. (2007). Vehicle Populsion System, Introduction to Modelling and Optimization. Springer Verlag, Zürich, 2 edition.

Hambley, A.R. (2005). Electrical Engineering, principles and applications. Pearson Education, Upper Sadle River, 3 edition.

Krysander, M., Åslund, J., and Nyberg, M. (2008). An efficient algorithm for finding minimal over-constrained sub-systems for model-based diagnosis. IEEE Trans. on SMC - Part A: Systems and Humans, 38(1).

Page, E. (1954). Continous inspection schemes. Biometrika, $41,100-115$.

Pulido, B. and Gonzalez, C. (2004). Possible conflicts: a compilation technique for consistency-based diagnosis. IEEE Trans. on SMC - Part B, 34(5), 2192-2206.

Sundström, C. (2011). Vehicle level diagnosis for hybrid powertrains. Technical report. Licentiate thesis LiUTEK-LIC-2011:27, Thesis No. 1488.

Svärd, C. and Nyberg, M. (2010). Residual generators for fault diagnosis using computation sequences with mixed causality applied to automotive systems. IEEE Trans. on SMC - Part A, 40(6), 1310-1328. 